\title{
TRADUCCIÓN Y DOBLAJE: ANÁLISIS DE FUCK \\ Y SU TRADUCCIÓN AL ESPAÑOL EN JACKIE BROWN \\ Betlem Soler Pardo \\ Universitat de València
}

\begin{abstract}
Four-letter words are the most obscene and vulgar words in the English language; these refer to parts of the body, bodily functions, human waste, and sex, to cite a few examples. Of all these categories, the one which involves sex is the most obscene of all, and among this group, the most vulgar is fuck (Montagu, 1967; Allan and Burridge, 2006). This paper aims, firstly, to examine a paradigmatic sample of the f-word as it appears in Jackie Brown, a neo noir film directed by Quentin Tarantino; secondly, to carry out research on the categorization of fuck based on the work of McEnery and Xiao (2004); thirdly, to analyse the translation for dubbing of this controversial word into Spanish; and finally, to establish if the presence of fuck in the Spanish version of Jackie Brown has decreased or increased after having carried out the transfer of words.
\end{abstract}

KEYWORDS: audiovisual translation, dubbing, swearwords, the f-word, Jackie Brown, Quentin Tarantino

\section{RESUMEN}

Las llamadas palabras de cuatro letras son consideradas las más obscenas y vulgares de la lengua inglesa, ya que hacen referencia a las partes del cuerpo, a las funciones fisiológicas, al producto de éstas, y al sexo, entre otras. De todas estas categorías, la concerniente al sexo es considerada la más obscena y, entre este grupo, la palabra más vulgar es fuck(Montagu, 1967; Allan and Burridge, 2006). Este artículo tiene como objetivo examinar, en primer lugar, una muestra paradigmática de la palabra fucktal y como aparece en la película Jackie Brown, un film neo noir dirigido por Quentin Tarantino; en segundo lugar, pretende llevar a cabo una investigación sobre la clasificación de dicha palabra basando nuestro estudio en el trabajo de McEnery y Xiao (2004); en tercer lugar, analizar la traducción para el doblaje de esta palabra al español y por último, establecer si la presencia de joder en la versión española ha disminuido o aumentado en la transferencia lingüística. 
PALABRAS CLAVE: traducción audiovisual, doblaje, lenguaje obsceno, f-word, Jackie Brown, Quentin Tarantino

\section{INTRODUCCIÓN}

Las llamadas four-letterwords o palabras de cuatro letras -la mayoría de las palabras malsonantes utilizadas de manera más frecuente en inglés suelen ser de cuatro letras, de ahí su nombre- son consideradas las expresiones más obscenas y vulgares de la lengua inglesa. Estas palabras pueden hacer referencia a las partes del cuerpo; a las funciones fisiológicas o al producto de éstas; y al sexo, entre otros aspectos. Por ser una mezcla entre aquello prohibido desde el punto de vista religioso, profano/blasfemo, y lo que se considera moralmente o socialmente inaceptable, obsceno, las palabras malsonantes han sido declaradas expresiones nocivas y perjudiciales a lo largo de la historia desde el punto de vista social $y$, por tanto, se nos ha exigido continuamente eliminarlas de nuestro vocabulario. Ambos conceptos, profano/blasfemo y obsceno, hacen que dichas palabras cobren más fuerza emocionalmente hablando y que se hayan convertido en expresiones de uso frecuente entre individuos de distintas edades: "The four-letterwords are stronger because they deliver a fusillade from both barrels at once, the profane and the obscene" (Montagu, 1967: 302). En definitiva, representan lo prohibido, lo tabú, entendiendo como tal aquella acción u objeto que puede causar ofensa o daño a otra persona, aquello que se entiende como "fuera de lugar" (Toledano, 2002: 220). Tales acciones han llegado incluso a estar prohibidas por ley en períodos concretos de la historia. En cierto modo, cualquier conducta podría considerarse tabú según las diferentes comunidades.

Por otra parte, de entre las palabras de cuatro letras, la categoría percibida como más obscena es la relacionada con el sexo y, dentro de ésta la palabra más obscena es fuck y derivados (Montagu, 1967: 303). El sexo ha sido siempre considerado tabú en la sociedad occidental tal y como expresa Jay: "Sexuality is one of the most tabooed aspects of human existente" (2000: 85). Por ello, los padres, tutores, y/o profesores han transmitido este sentido de prohibición a sus hijos y alumnos para que lo aprendan desde una edad temprana y entiendan que el sexo es tabú y, por tanto, que deben aplicar una serie de restricciones al respecto. Por ejemplo, de niños ya se nos prohíbe usar expresiones relacionadas con el sexo y, como resultado, estas palabras se han transformado en ofensivas y con ellas el sexo en sí. Sin embargo, el sentido original de la palabra como referencia al acto sexual o al sexo ha variado con el tiempo, y a este sentido se han unido otros significados en el transcurso de los años, tal y como veremos en los ejemplos seleccionados para este estudio. Así 
pues, aunque la palabra fuck tradicionalmente recordara cualquier aspecto sexual, hoy en día dicha expresión ya no es interpretada en su sentido más literal sino que se reconoce en ella una palabra malsonante que se puede utilizar en varios contextos y que no está condicionada únicamente al acto sexual. Respecto a su aceptación, aunque su uso escrito sea restringido y su uso hablado sea bastante limitado en público, como podemos comprobar a través del cine, hoy en día fuck ha pasado de ser una palabra impronunciable a ser una palabra de uso casi frecuente, como indicábamos anteriormente, aunque con ciertas restricciones. Esto se hace evidente en películas como las de Tarantino -y en especial en Jackie Brown - en las que los insultos de carácter sexual superan al resto de categorías (escatología, insultos relacionados con la prostitución o con las funciones fisiológicas, etc.).

Jackie Brown es la adaptación para la gran pantalla de la novela de Elmore Leonard titulada Rum Punch (1992). Aunque Tarantino cambia la violencia visual de sus anteriores películas -PulpFictiony ReservoirDogs- por sofisticación en Jackie Brown, sigue manteniendo el sentido del humor y el lenguaje verbal que lo caracteriza. Con respecto a la trama, la Srta. Brown es una azafata de vuelo que intenta introducir dinero en los EEUU de manera ilegal y que, a su vez, intenta aprovecharse de sus compañeros para apropiarse de estas grandes cantidades. Mafiosos, policías corruptos y asesinos son los protagonistas principales de este largometraje en el que el fatalismo y la ambigüedad moral se encuentran presentes; por lo tanto y debido a su temática, Jackie Brown alienta los insultos sexuales y sobre todo el uso de la palabra fuck, ya que se trata de un film noir con personajes "amorales, materialistas, perdedores dispuestos a cambiar su suerte, seres ajenos a unos destinos que no pueden controlar" (Comas, 2005: 203). Siguiendo las características del noir, en el film aparecen protagonistas fracasados que están dispuestos a dar un giro drástico a sus vidas, y personajes codiciosos y cínicos sin sentido de la moral, en definitiva, personajes inclinados hacia la violencia verbal.

\section{METODOLOGÍA}

La metodología utilizada para esta investigación consistió en recopilar un corpus de los insultos sexuales que aparecían en la versión original en inglés de Jackie Brown. Una vez llevada a cabo esta tarea, centramos nuestra atención en aquella palabra que se repetía de manera más frecuente dentro de esta clasificación, f-word y derivados, y decidimos realizar un análisis exhaustivo de ésta y compararla con la versión doblada al español. De esta manera se podrían observar las posibles diferencias y similitudes entre la obra original y la versión doblada. Para el análisis, en un principio tomamos como referencia la obra 
publicada en la editorial Faber and Faber pero al comprobar que los guiones publicados no contemplaban los insultos al completo, decidimos tomar como referencia los diálogos orales de la película.

La causa de esta omisión en el texto publicado puede deberse a que el número de tacos tiende a ser reducido en los textos escritos para evitar un posible conflicto (Díaz Cintas, 2001: 130) y, por lo tanto, justificaría la existencia de un menor número de palabras malsonantes en el registro escrito el guión publicado por la editorial- que en la pista sonora de la película original.

\section{ANÁLISIS Y RESULTADOS}

\subsection{ANÁlisis y CATEgORIZACIÓN DE LOS INSUltos SEXUALES EN JACKIE} BROWN

Jackie Brown es, en términos de cantidad, la película más obscena de Tarantino - 339 insultos de los cuales 96 son de carácter sexual (Soler Pardo, 2012). Estos 96 insultos sexuales se encuentran monopolizados por la palabra fuck y derivados durante los 151 minutos que dura de la película. Tarantino utiliza estos insultos constantemente para expresar enfado, ira, sorpresa, y para recalcar una idea o enfatizar una expresión, como también apunta Pujol (2006) en un estudio similar sobre el uso de los insultos en Tarantino.

Con el fin de analizar los insultos sexuales que aparecen en Jackie Brown, he establecido un criterio de clasificación para la palabra fuck basándome en el trabajo de McEnery y Xiao (2004) para quienes el término fuck puede usarse como: (1) insulto general; (2) insulto personal destinado a una entidad definida; (3) taco; (4) con un uso determinado; (5) uso literal que denota un referente tabú; (6) intensificador enfático; (7) forma pronominal; (8) frase idiomática; y (9) metalingüístico o inclasificable debido a la falta de contexto. Así pues, en vista de los resultados obtenidos tras realizar el estudio, he tomado como referencia las cuatro estructuras del modelo de McEnery y Xiao, que más se repetían en la obra de Tarantino, de manera que los insultos que aparecen en Jackie Brown se clasificarían según: (1) su sentido literal; (2) si actúan como interjecciones; o (3) como intensificadores enfáticos; y (4) cuando se usan como insulto general.

Observemos más claramente las cifras completas en la siguiente tabla:

\begin{tabular}{|l|l|}
\hline Clasificación de Joder & JB \\
\hline Sentido Literal & 4 \\
\hline Intensificador Enfático & 63 \\
\hline Insulto General & 26 \\
\hline
\end{tabular}




\begin{tabular}{|l|l|}
\hline Interjección & 3 \\
\hline & \\
\hline Total & $\mathbf{9 6}$ \\
\hline
\end{tabular}

Figura 1. Clasificación de "fuck"

Como se puede apreciar en la figura 1, en Jackie Brown, el uso de fuck/fucking como intensificador enfático es estadísticamente más significativo si lo comparamos con los otros tres usos estudiados en la clasificación. Por consiguiente, el sentido literal de fuck habría quedado relegado a un penúltimo lugar. Observemos el porcentaje total en la siguiente figura:

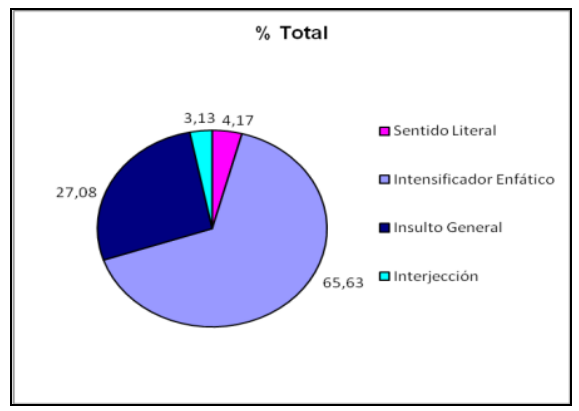

Figura 2. Porcentaje total de Fuck/fucking clasificado según su sentido literal, intensificador enfático, insulto general o interjección

Las cifras expuestas en las figuras 1 y 2 indican que la palabra fuck ha perdido su sentido literal -copular- en Jackie Brown, y se utiliza como intensificador enfático en la mayoría de los casos en oraciones que no hacen alusión a su carácter sexual original. Esta cifra representa el 65,63\% de todos los insultos sexuales, y corresponde concretamente a 63 ejemplos. La segunda situación en la que se emplea de manera más frecuente es como insulto general, cuya cifra ocupa el $27,08 \%$ del total y corresponde a 26 ejemplos. Aunque es una cifra importante, se aleja significativamente del porcentaje anterior. La palabra fuck se emplea en el largometraje haciendo referencia a su significado literal en un 4,17\% de las situaciones, lo que asciende a un total de 4 insultos; un porcentaje que se podría considerar extremadamente reducido y, si más no, curioso, si se parte de la base de que el sentido original de la palabra fuck es el de copular. Por último, tanto fuck como fucking actúan como interjecciones en un $3,13 \%$ lo cual supone una cifra de 3 ejemplos a lo largo de la película. A continuación presentamos una tabla donde se expone una muestra representativa de 10 ejemplos que intercalan las cuatro situaciones expuestas en la figura 1: 


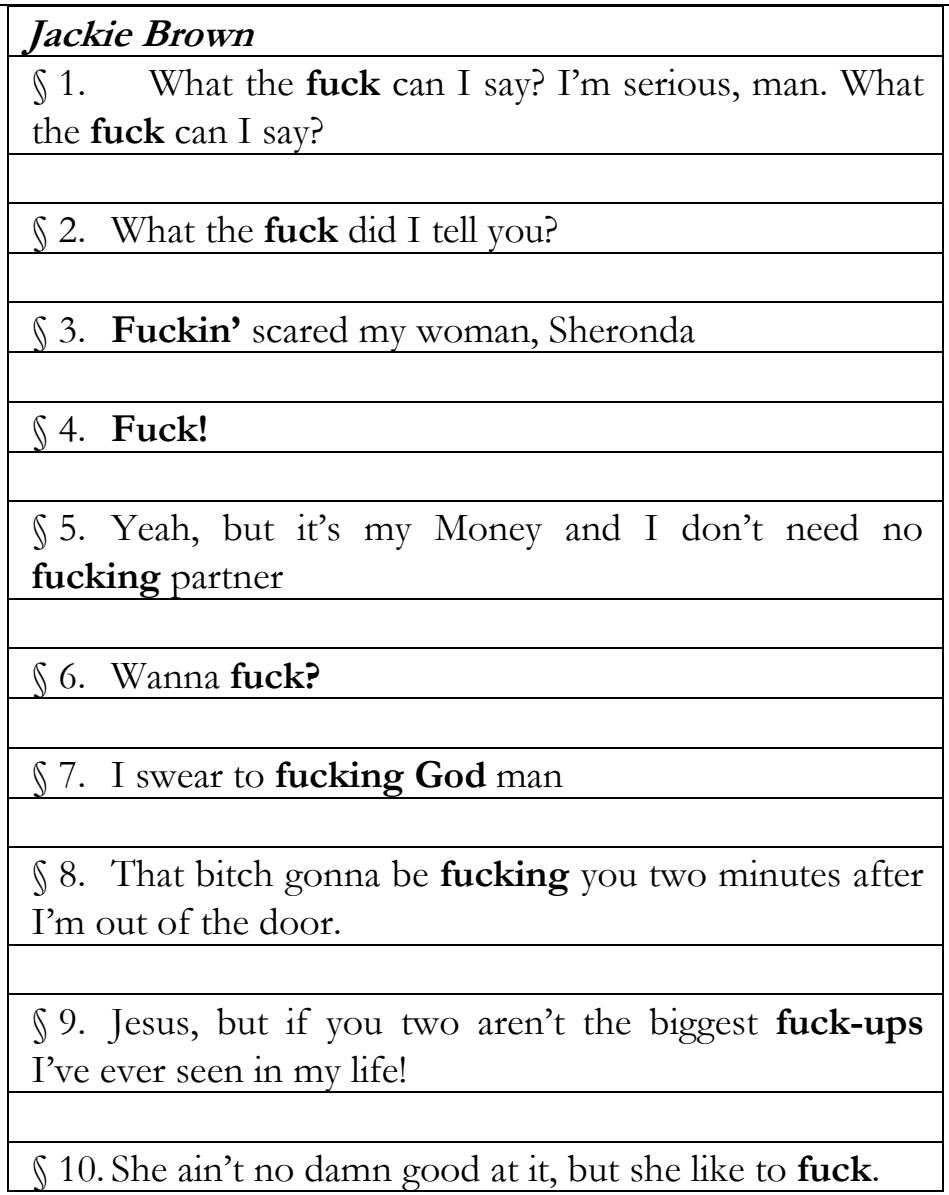

Figura 3. -"Ejemplos de fucken Jackie Brown-"

En la figura 3, podemos ver el primer ejemplo de Jackie Brown en el cual fuck es utilizado como intensificador enfático. El ejemplo $\$ 2$ presenta una estructura similar e igualmente funciona como intensificador enfático. En el siguiente ejemplo \$3, fucking aparece como un enfatizador que modifica a un verbo, y en el número $₫ 4$ fuck actúa como interjección. El número $₫ 5$ contiene la palabra fucking que actúa como un intensificador enfático que se encuentra modificando a un sustantivo. En el ejemplo número $\$ 6$, fuck es empleado en su sentido literal, copular. El número $\$ 7$ presenta un ejemplo perteneciente al grupo de insultos religiosos utilizado con el propósito de intensificar la oración. El siguiente ejemplo, $§ 8$, fucking es utilizado es su sentido literal, y el penúltimo ejemplo actúa como insulto general. Finalmente, en el último ejemplo en Jackie Brown, $\$ 10$ se emplea fuck en el sentido sexual del término. 
Como consecuencia de la cuantía de tacos que aparecen en las películas de Tarantino, su traducción al español resulta en una lucha constante entre la fidelidad al texto y lo que resultaría (in)apropiado para cierto tipo de audiencia. En la siguiente sección, analizaremos el doblaje al español de los 10 ejemplos presentados en el apartado 3.1, para comparar si la traducción al español ha sufrido algún tipo de modificación que haya que resaltar respecto al número de insultos traducidos:

\begin{tabular}{|c|c|}
\hline \multicolumn{2}{|l|}{ Jackie Brown } \\
\hline $\begin{array}{l}\int 1 . \text { What the fuck can I say? } \\
\text { I'm serious, man. What the fuck } \\
\text { can I say? }\end{array}$ & No sé qué decir, tío. \\
\hline $\begin{array}{l}\int 2 . \\
\text { you? }\end{array}$ & $\begin{array}{l}\text { Yo no te he dicho que te cargues } \\
\text { a nadie. }\end{array}$ \\
\hline $\begin{array}{l}\text { S } 3 . \quad \text { Fuckin' scared my } \\
\text { woman, Sheronda }\end{array}$ & $\begin{array}{l}\text { Le pegaron un susto de muerte a } \\
\text { Sheronda, mi mujer. }\end{array}$ \\
\hline \4. Fuck! & Joder, coño, quéasco! \\
\hline $\begin{array}{l}\int 5 . \quad \text { Yeah, but it's my Money } \\
\text { and I don't need no fucking } \\
\text { partner }\end{array}$ & $\begin{array}{l}\text { Sí, pero es mi dinero y no } \\
\text { necesito una socia. }\end{array}$ \\
\hline \$6. Wannafuck? & ¿Quieresfollar? \\
\hline $\begin{array}{ll}\int 7 . & \text { I swear to fucking God } \\
\text { man } & \end{array}$ & Lo juro por Dios. \\
\hline $\begin{array}{l}\int 8 . \text { That bitch gonna be } \\
\text { fucking you two minutes after } \\
\text { I'm out of the door. }\end{array}$ & $\begin{array}{l}\text { Se te habrá follado dos minutos } \\
\text { después de que yo me fuera. }\end{array}$ \\
\hline $\begin{array}{l}\int 9 . \text { Jesús, but if you two } \\
\text { aren't the biggest fuck-ups I've } \\
\text { ever seen in my life! }\end{array}$ & $\begin{array}{l}\text { Jesús, Ordell y tú sois la pareja de } \\
\text { chapuzas más grande que he visto } \\
\text { en mi vida. }\end{array}$ \\
\hline
\end{tabular}


$\int 10$. She ain't no damn good at it, Lo hace de puta pena, pero le but she like to fuck.

Figura 4. "Traducción al español de fuck/fuckingen Jackie Brown"

En el primer ejemplo de la figura 4, podemos observar la frase what the fuck utilizado como intensificador enfático. Cuando se encuentra en esta posición la mejor solución consiste en traducirla por "joder" y situarla al principio o al final de la oración. En este caso, una posible solución sería sustituir "joder" por "tío" para conservar el tono inicial o, traducir fuck por “coño”, de manera que resultaría: “¿Qué coño puedo decir, tío?”.

Algo similar ocurre en el ejemplo $\ 2$, así pues, una solución sería añadir también "joder" a la oración o sustituirla por "coño" para añadir el tono que se pretende en el original: "¿Pero yo qué coño te he dicho, eh?”.

En el siguiente ejmplo, §3, fucking aparece modificando a un verbo, lo cual hace que su traducción al español sea complicada. Se podría conseguir un mejor resultado si añadiéramos "joder" o "coño" al final de la oración.

El ejemplo número $\int 4$ no presenta mayor dificultad ya que podría haberse traducido por "jodido", de manera que quedaría: "La policía te ha jodido la mente/coco". Esta palabra puede presentar confusión ya que no se trata de una expresión genuina, sino que se trataría más bien de un calco del inglés $y$, por lo tanto, le faltaría naturalidad. De manera que estaríamos hablando del "síndrome de bipolaridad lingüística" tal y como señalan Antonini \& Chiaro (2009: 11). El síndrome de bipolaridad lingüística consiste en ser consciente de que la lengua usada para el doblaje podría no ser la lengua empleada en el día a día y, a pesar de ello, que los espectadores lo acepten con normalidad.

En el siguiente ejemplo, $\sqrt{5}$, fucking modifica a un sustantivo y podría traducirse como "puta", por lo tanto, quedaría de la siguiente manera: "puta compañera". Otra posible solución sería añadir "joder" al final de la oración para conseguir un resultado similar.

En el ejemplo número $\$ 6$, podemos ver un insulto compuesto fucking around usado en gerundio que ha sido traducido como "movimiento brusco". Una posible expresión que podría ser usada en este contexto sería "intentar joder a alguien"; así pues la oración quedaría de la siguiente manera: "No intentes joderme". Sin embargo, el adaptador podría encontrarse con el problema de que la frase en español fuera más corta y, quizás, debería encontrar otra alternativa para solucionar el problema del sincronismo labial.

El ejemplo $\$ 7$ incluye un insulto que pertenece a la categoría sexual y religiosa fucking God. Aunque la blasfemia es un modo de insulto bastante común en el Estado Español, al haber sido un país fervientemente religioso, el 
maldecir usando este tipo de expresiones religiosas puede causar sorpresa al telespectador si añadiéramos puto en el mismo contexto. Por ello, quizás, el traductor ha dejado ese taco sin traducir en esta frase. Si se hubiera llevado a cabo la transferencia lingüística, el resultado de la clasificación de la película sería más alta y, además, podría causar descontento entre ciertos grupos religiosos.

El ejemplo $\int 8$ muestra al phrasal verb fuck up el cual podría haberse traducido como "jode” o "jode vivo" para adquirir más énfasis: "Pero el estar fichado te jode vivo y Jackie no pudo entrar en ninguna compañía importante”.

El penúltimo ejemplo, $\$ 9$ fuck-ups ha sido traducido inocentemente como "chapuzas", así pues, podría haberse encontrado una mejor equivalencia con, por ejemplo, expresiones del tipo "putos desastres" o en el singular "puto desastre".

El último ejemplo presentado para este artículo, $\$ 10$, sitúa al traductor en una posición difícil una vez más. Por lo tanto, sugeriría traducir shut the fuck up como "cierra la puta boca y no te muevas (ni un pelo)".

En definitiva, la lengua española nos ofrece varias opciones para traducir fuck y derivados: mierda (de); joder; coño; puto/a; y jodido. Y dependiendo del contexto léxico y sintáctico se emplearían unos u otros términos. Algunas de estas opciones han sido utilizadas en la traducción de Jackie Brown al completo, y otras han sido omitidas en su totalidad debido a ciertas restricciones. Respecto a si el nivel de insultos en español ha disminuido, aumentado o se ha mantenido, podemos afirmar que, después de realizar un análisis exhaustivo de la traducción para el doblaje de los insultos en Jackie Brown en otro trabajo anterior (Soler, 2012), se pudo comprobar que de los 96 ejemplos sexuales - fuck y derivados- 42 no han sido traducidos o han sido suavizados, tal y como se puede observar en el siguiente gráfico:

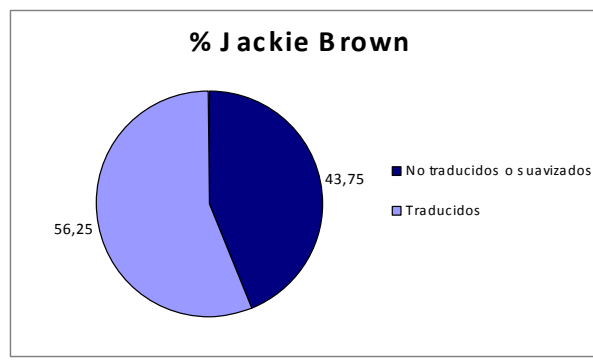

Figura 5. "Porcentaje de insultos traducidos"

En vista de tales resultados, podemos constatar que el nivel de insultos en la traducción al español del cuarto largometraje del director Quentin 
Tarantino es inferior al original, ya que el $43,75 \%$ de los insultos han sido traducidos de manera imprecisa o han sufrido algún tipo de omisión o modificación (Soler Pardo, 2012); según estos datos, el porcentaje de insultos no traducidos sería casi equiparable a la de insultos traducidos tal y como se aprecia en el gráfico. La elevada cifra de tacos sin traducir nos lleva a concluir que existen varios factores que han sido aplicados al realizar la traducción de la película. Por tanto, una vez analizada y establecida una correspondencia entre la traducción de la palabra fuckpara el doblaje de Jackie Brown al español, y en vista del descenso en el número de insultos una vez llevada a cabo la transferencia lingüística, podemos extraer una serie de conclusiones que nos llevarían a explicar los porqués de dicho descenso:

(1) La falta de espacio/tiempo. Ya que la estructura sintáctica del español es necesariamente más larga que la del inglés, y por lo tanto, al tener que eliminar, para que coincidan los textos, algún tipo de información se decide prescindir la que no aporta un significado importante a la oración, los tacos. Díaz Cintas (2001), en su estudio sobre la traducción audiovisual, y José Llurba, traductor y adaptador de textos audiovisuales, en una entrevista realizada en el 2003 señala: "Si es un taco superficial que no aporta nada a la frase es lo primero que se elimina" (Llurba en Del Águila y Rodero 2005: 47). Llurba también señala que el traductor no es el encargado de eliminar los tacos sino que es una tarea llevada a cabo por el adaptador/ajustador: "El suavizarlo (el taco) más bien es cuando llega a la adaptación, que es el adaptador quien no tiene espacio para meter los tacos" (ibidem);

(2) Sincronización labial: el adaptador tendrá que realizar los cambios necesarios para conseguir sincronizar el movimiento labial en cuanto sea posible en la traducción al español. Y entonces, cuando esto no es viable, algunas palabras tienden a ser eliminadas para conseguir un sincronismo (casi) perfecto, como por ejemplo, los insultos;

(3) La decisión de la distribuidora de mutilar el texto con fines comerciales, es decir, reescribir el texto audiovisual para que la clasificación sea más baja y tenga más éxito comercial. Por ejemplo, como advierte Díaz Cintas (2001), la distribuidora "Buena Vista" pide al traductor/adaptador que cualquier traducción les sea entregada libre de "lenguaje obsceno", para obtener el certificado para un grupo en concreto y también señala que, aunque no existe ninguna ley que lo estipule, el adaptador se siente "obligado" a hacer lo que le exigen (ibídem 47), algo que también señalan otros expertos en traducción:

En ocasiones existen clientes que solicitan al ajustador que se supriman, por ejemplo, los tacos para de este modo obtener la autorización para una determinada edad, por lo que el ajustador se ve limitado en su trabajo por una serie de condicionantes. En estas situaciones, suele aceptar las condiciones, aunque, 

una película (Del Águila y Rodero, 2005: 47).

(4) Otra posible contrariedad es la tradición de un país conservador: la moral del ajustador que, al no ser revisado su trabajo en profundidad, podría permitirse cierta licencia y omitir aquello que no le pareciese moralmente correcto. Así pues, dominaría la idea de que la televisión y el cine tienen que ser ejemplo de moralidad ética y lingüística, es decir con la auto-censura se asegurarían una mayor audiencia y reconocimiento.

\section{CONCLUSIÓN}

En suma, después de haber llevado a cabo un análisis de la palabra fuck en Jackie Brown podemos concluir que dicha palabra se utiliza a lo largo del film en sentido literal, como intensificador enfático, como insulto general, y como interjección. Además, podemos concluir que se podría haber restringido el uso de algunos insultos al realizar la traducción lingüística en base a los argumentos mencionados en la sección anterior (3.2.), con la excepción de la palabra fuck utilizada en su sentido sexual, en cuyo caso, la traducción al español se habría realizado sin omisiones.

En definitiva, traducir un texto audiovisual es una tarea complicada de la que no se consiguen resultados definitivos, sino que se obtienen nuevas perspectivas y se crean opiniones diversas. Si a esto le añadimos la traducción del lenguaje sexual comprobaremos que se trata de una tarea que ha estado siempre estrechamente debatida, y que se ha apoyado en diversos factores dependiendo de la época, las circunstancias políticas y la ideología del traductor. No cabe duda de que el punto de vista del traductor es un factor a tener en cuenta a la hora de traducir un texto, ya que es difícil permanecer imparcial cuando se utiliza una serie de palabras o expresiones. Esto es precisamente lo que ocurre con el lenguaje obsceno en general y es también uno de los factores adicionales, pero no el único, en Jackie Brown ya que la falta de espacio/tiempo y la sincronización labial desempeñan un papel primordial en la traducción audiovisual.

Además, si partimos de la base de que los insultos siempre han tendido a considerarse ofensivos, pensaríamos que éstos podrían ser eliminados de nuestro vocabulario sin ninguna consecuencia. Sin embargo, estas palabras en particular son una pieza importante del idioma y no resulta sencillo eliminarlas sin que se produzca un cambio, ya que, en relación con Tarantino, los tacos funcionan como determinantes sociales, raciales y culturales y aportan una información única como, por ejemplo, el nivel socio-cultural del personaje tal y 
como ocurre en este su cuarto film. Tarantino utiliza la violencia física en Jackie Brown como leitmotiv, lo cual justifica la violencia verbal presente en la película. El hecho de que ésta sea su obra más obscena -339 insultos- es un factor estrechamente relacionado con el hecho de que en ella aparezcan antihéroes: ladrones, gánsteres, atracadores de bancos, criminales que no tienen éxito en su misión y lo expresan a través de palabras que denotan ira y desesperación. Dada la descripción de dichos personajes, podemos afirmar que, al no ser un ejemplo a seguir, el espectador no desea imitarlos, ni parecerse a ellos, no los admira $y$, por tanto, a estos personajes se les permite usar tacos en la versión original. Por lo tanto, el hecho de que se hayan eliminado insultos en la traducción de Jackie Brown nos conduce a pensar que no se ha considerado el contexto socio-cultural; y estudiar la traducción de manera aislada sin tener en cuenta este aspecto supone una restructuración del producto final que, sin duda, altera el sentido original de la película.

\section{BIBLIOGRAFÍA}

Allan, KeithyBurridge, Kate (2006): Forbidden Words: Taboo and the Censoring of Language. Cambridge, Cambridge University Press.

Antonini, R. Y Chiaro, D. (2009): “The Perception of Dubbing by Italian Audiences" en Díaz Cintas, J. y Anderman, G. (eds.): Audiovisual Translation: Language Transfer on Screen. Basingstoke, Palgrave MacMillan.

Comas, Ángel (2005): De Hitchcock a Tarantino. Madrid, T\&B Editores.

Del Águila, Ma E. Y Rodero ANTÓn, E. (2005): El proceso de doblaje take a take. Salamanca, Universidad Pontificia de Salamanca.

DíAz CinTAS, JORGE (2001): La traducción audiovisual: El subtitulado. Salamanca, Ediciones Salmar.

Jay, Timothy (2001): Why We Curse: A Neuro-Psycho-Social Theory of Speech. Philadelphia/Amsterdam, John Benjamins Publishing Company.

MCEnERY, A. Y XiAO, Z. (2004): "Swearing in modern British English: The Case of Fuck in the BNC", Language and Literature 13/3, 235-268.

Montagu, Ashley (1967): The Anatomy of Swearing. New York/London, Collier-Macmillan.

SOlER PARdo, BETLEM (2012): Swearing and Translation: A Study of the Insults in the Films of Quentin Tarantino. Valencia, Servei de Publicacions de la Universitat de València.

TARANTINO, Quentin (1998): Jackie Brown. London, Faber and Faber Limited. PujOL, DíDAC (2006): "The 'Translation and Dubbing of 'Fuck' into Catalan: The Case of From Dusk till Dawn", The Journal of Specialised Translation, 6, 121-133. 
TOLEDANO BUENDÍA, CARMEN (2002): "Algunas consideraciones generales sobre la traducción, la obscenidad y la traducción de la obscenidad", Revista Canaria de Estudios Ingleses, 44, 217-232.

\section{FILMOGRAFÍA}

ReservoirDogs. D: Quentin Tarantino. P: Lawrence Bender, Harvey Keitel. SC: Q. Tarantino, Roger Avary. Du: 102' Live Entertainment, 1992.

PulpFiction. D: Quentin Tarantino. P: Lawrence Bender. SC: Q. Tarantino, Roger Avary. Du: 154'. Miramax Films, 1994.

Four Rooms: The Man from Hollywood. D: Quentin Tarantino. P: Lawrence Bender, Heidi Vogel, Paul Hellerman, Scott Lambert. SC: Q. Tarantino. Du: 97' (whole film) Color. A Band Apart-Miramax, 1995.

Jackie Brown. D: Quentin Tarantino. P: Lawrence Bender, Paul Hellerman. SC: Q. Tarantino (adapted from Elmore Leonard's novel Rum Punch). Du: 151'. Color.Lawrence Bender Productions-A Band Apart-Miramax- Mighty Afrodite Productions, 1997.

Kill Bill vol I. D: Quentin Tarantino. P: Lawrence Bender. SC: Q. Tarantino. Du: 111'. A Band Apart-Miramax, 2003.

Kill Bill vol. II. D: Quentin Tarantino. P: Lawrence Bender. SC: Q. Tarantino. Du: 136'. A Band Apart-Miramax, 2004.

Grindhouse: Death Proof. D: Quentin Tarantino. P: Elizabeth Avellan, Robert Rodríguez, EricaSteinberg and Quentin Tarantino. SC: Q. Tarantino. Du: 95'. The Weinstein Company, 2007.

Inglorious Basterds. D: Quentin Tarantino. P: A Band Apart-A ZehnteBabelsberg Film GmbH. SC: Q. Tarantino. Du: 146'. Universal-The Weinstein Company, 2009. 